\title{
Método de compresión de electrocardiogramas basado en muestreo compresivo
}

\author{
Rodolfo Moreno-Alvarado ${ }^{1}$, Héctor Pérez-Meana ${ }^{2}$, \\ Mariko Nakano-Miyatake ${ }^{2}$, Daniel Robles-Camarillo ${ }^{1}$ \\ ${ }^{1}$ Universidad Politécnica de Pachuca, Zempoala, Hidalgo, \\ México, \\ ${ }^{2}$ Instituto Politécnico Nacional, Esime Culhuacan, \\ México \\ rg.moreno@lasallistas.org.mx., \\ \{hmperezm, mnakano\}@ipn.mx, danielrc@upp.edu.mx
}

\begin{abstract}
Resumen. El muestreo compresivo (MC) es un enfoque prometedor que ayuda a comprimir y recuperar señales de electrocardiograma (ECG). Esta técnica explota la dispersión y la compresibilidad de las señales de ECG con una representación dispersa en cierto dominio $\Psi$ usando la matriz de medición $\Phi$, que satisface la propiedad de isometría restringida. El método propuesto combina primero la dispersión y la compresibilidad de las señales de ECG en el dominio de la Transformada Wavelet Discreta (DWT) para lograr una señal dispersa y compresible. En segundo lugar, utilizando una matriz de medición ortogonal (MMO) basada en Transformada de Coseno Discreta (DCT) tomamos medidas incoherentes y finalmente se mejora el proceso de recuperación usando la traspuesta de (MMO) en vez de los algoritmos de reconstrucción de MC. Los resultados experimentales utilizan la base de datos de arritmias MIT-BIH mostrando que pueden obtenerse ganancias significativas, en términos de la tasa de compresión y calidad de reconstrucción, mediante el algoritmo propuesto en comparación con los métodos de MC actuales.
\end{abstract}

Palabras clave: Electrocardiograma, muestreo compresivo, coherencia, dispersión.

\section{Electrocardiogram Compression Method Based on Compressive Sampling}

\begin{abstract}
Compressive sampling (CS) is a promising approach which aids to compress and recover electrocardiogram (ECG) signals. This technique exploits the sparseness and compressibility of ECG signals with sparse representation in
\end{abstract}




\begin{abstract}
certain domain $\Psi$ using measurement matrix $\Phi$ which satisfies the restricted isometry property. The proposed method first combines the sparseness and compressibility of ECG signals in Discrete Wavelet Transform (DWT) domain to achieve a sparse and compressible signal, secondly using an orthogonal measurement matrix (OMM) based on Discrete Cosine Transform (DCT) we take incoherent measurements and finally improve the recovery process, with the OMM transpose instead the use of typical reconstruction algorithms for CS. Experimental results utilizing the MIT-BIH Arrhythmia Database show that significant performance gains, in terms of compression rate and reconstruction quality, can be obtained by the proposed algorithm compared to current CS methods.
\end{abstract}

Keywords: Electrocardiogram, compressive sampling, coherence, sparsity.

\title{
1. Introducción
}

Estudios de la OMS reportan a las Enfermedades cardiovasculares (ECV) como la principal causa de muerte en todo el mundo. Se calcula que en 2012 murieron por esta causa 17,5 millones de personas, lo cual representa un $31 \%$ de todas las muertes registradas en el mundo. Para las personas con ECV o con alto riesgo cardiovascular (debido a la presencia de factores de riesgo, como la hipertensión arterial, la diabetes o la hiperlipidemia) son fundamentales la detección y el tratamiento temprano [1]. Así, a fin de detectar y realizar un diagnóstico clínico temprano de forma no invasiva de las ECV se usa el ECG, el cual, nos revela información importante sobre el estado del corazón por lo que es considerado un estándar en el diagnóstico de arritmias.

Debido a que el registro del ECG se lleva a cabo mediante sistemas de monitoreo, estos, deben de ser capaces de manejar una gran cantidad de datos, almacenarlos, procesarlos y en algunos casos transmitirlos, ya sea a otro sistema o al profesional de la salud encargado para su evaluación, por tal motivo, es de gran importancia la compresión del registro del ECG generado.

Actualmente, aunque se tiene un auge en los métodos de compresión y son diversos, es necesario desarrollar algoritmos de compresión de ECG que produzcan mejores relaciones de compresión y una mayor calidad en los datos que son reconstruidos. El ya conocido enfoque tradicional de compresión y reconstrucción de señales o imágenes a partir de datos medidos sigue al teorema de muestreo de Shannon-Nyquist WhittakerKotelnikov, el cual establece que la frecuencia de muestreo debe ser al menos del doble de la frecuencia más alta presente en la señal [8]. Del mismo modo, el teorema fundamental del álgebra lineal sugiere que el número de muestras recolectadas (mediciones) presentes en una señal con dimensión finita debe ser al menos tan grande como su longitud (dimensión) para garantizar la reconstrucción.

En los últimos años se ha venido gestando una nueva teoría del muestreo, la teoría del MC [3], que, por su parte, permite la reconstrucción de señales a partir de menos muestras (mediciones) o datos; que las sugeridas por el muestreo convencional. Esta novedosa teoría propuesta por Donoho [8] y Candes [3] proporciona un enfoque 
fundamentalmente nuevo para la adquisición y compresión de datos simultáneamente [4].

En comparación con los algoritmos de compresión de ECG convencionales, el MC tiene algunas ventajas importantes: entre las cuales se encuentra la transferencia comprimida y codificada de la señal de interés [12] y la fácil integración de codificadores en hardware por mencionar algunas. Así, el MC va teniendo un gran auge en la rama de ECG, existen diferentes trabajos que implementan de manera formidable cada vez más esta técnica: Desde la implementación de sistemas dedicados [5, 14], compresión de datos [5], transmisión [7], arquitectura de codificadores [16], matrices de medición [16, 17], medidas contra el ruido [18], y la reconstrucción eficiente de los coeficientes de baja magnitud [19, 21], entre otros. Sin embargo, a pesar de los avances, todos los trabajos mencionados anteriormente estos, utilizan algoritmos de reconstrucción que incrementan el costo computacional en su implementación.

En este trabajo se propone un método de compresión basado en muestreo compresivo, el cual tiene como objetivo la recuperación de la señal de ECG a partir de la traspuesta de la matriz de medición generada, sin realizar iteraciones como en ciertos algoritmos de reconstrucción de MC. A lo largo de este proceso se usa la Transformada de Wavelet Discreta (DWT) así como la Transformada Inversa de Wavelet Discreta (IDWT) con el fin de dispersar, comprimir y recuperar nuestras señales de ECG. El rendimiento de este método de compresión propuesto se mide en términos del radio de compresión (CR) y la calidad de la señal reconstruida usando el porcentaje de diferencia cuadrático medio (PRD), así como el porcentaje de diferencia cuadrático medio normalizado (PRDN) los cuales se evalúan utilizando los registros extraídos de la base de datos de arritmias del MIT-BIH [15].

Los resultados de PSNR y NPSNR validan el algoritmo propuesto en comparación con el algoritmo presentado en [20]. El resto del documento está organizado de la siguiente manera. La Sección 2 presenta el Marco Teórico del MC. La Sección 3 detalla la dispersión de la señal de ECG, la construcción de la matriz de muestreo y las medidas utilizadas para la evaluación de los resultados. La Sección 4 presenta la metodología desarrollada. La Sección 5 presentan los resultados de la simulación. La Sección 6 presenta la Discusión y las principales conclusiones respectivamente.

\section{Muestreo compresivo}

Esta sección comprende los conceptos básicos del muestreo compresivo. Como ya se ha mencionado anteriormente, el MC es una técnica que permite reconstruir una señal a partir de pocas mediciones, es importante establecer que esta teoría se basa en dos principios: La dispersión y la incoherencia [3]. Dado que una señal puede ser representada bajo diferentes bases, se dice que está cumple con la condición adecuada de dispersión si en esta representación cuenta con un número pequeño de elementos $k$ distintos de cero, siendo esta condición fundamental para las señales compresibles. Por otro lado, la propiedad de incoherencia está inversamente relacionada con la coherencia, medida que indica la correlación que se tiene entre los elementos de la matriz de medición y la matriz o base en la cual se desarrolló la dispersión de la señal. 
Por lo anterior, es posible expresar el MC como un problema inverso, ver ecuación (1), donde en el contexto de los principios antes mencionados $x \in R^{N}$ es el vector $N \times$ 1 que representa a la señal de ECG en el dominio disperso y a su vez $\Phi$ representa la matriz de medición (el sensor que adquiere la información), así podemos denotar a las muestras de la señal comprimida como $z \in R^{M x N}$ donde $M<N$ :

$$
z=\Phi \mathrm{x} .
$$

Una vez que se tiene la señal comprimida $z$, esta se envía al receptor donde se recupera y decodifica por medio de la matriz $\Phi$ y los algoritmos de recuperación propuestos. Para recuperar con éxito la señal de ECG se requiere que $x$ sea dispersa, en el caso en el que $x$ no sea dispersa se busca una matriz de dispersión $\Psi$ de tal forma que $x$ pueda ser representada con pocos elementos. En general, $x=\Psi \alpha$ se construye usando distintas bases, en este trabajo, se considera como bases de dispersión a funciones wavelet.

Entonces, un algoritmo de MC puede recuperar $\alpha$ usando las mediciones disponibles $z$ y la matriz $\Theta=\Phi \Psi$, modelo que ha sido ampliamente utilizado para señales de ECG $[12,14]$. Por lo tanto, reescribiendo la ecuación (1) esta queda en términos de los coeficientes de señal dispersos $\alpha$ como:

$$
z=\Phi_{\mathrm{X}}=\Phi \Psi \alpha=\Theta \alpha
$$

Cabe mencionar que el proceso de medición no es adaptativo, lo que significa que $\Phi$ es fijo y no depende de la señal $x$, como se puede apreciar, es necesario construir una matriz $\Phi$ de tal forma que a pesar de reducir la dimensión de $x \in R^{N}$ a $z \in R^{M}$ la información esencial contenida en la señal $x$ no sea distorsionada o corrompida por este proceso. Una forma en la cual la matriz $\Phi$ pueda realizar este proceso es cumpliendo la propiedad de isometría reservada [4] ecuación (3). Donde se muestra que la constante de isometría reservada $\delta_{s}$ de la matriz $\Phi \in R^{m x N}$ se define como la más pequeña si se cumple para toda $x \in R^{N}$ en el espacio disperso $\mathrm{S}$ :

$$
\left(1-\delta_{s}\right)\|x\|_{2}^{2} \leq\|\Phi x\|_{2}^{2} \leq\left(1+\delta_{s}\right)\|x\|_{2}^{2} .
$$

Así, una matriz $\Phi$ satisface la propiedad de isometría reservada si $\delta_{s}$ es pequeña, otra condición relacionada se denomina coherencia, y fue introducida al MC en [3]. Para ser formal, la coherencia está definida por el máximo valor absoluto de la correlación cruzada entre las columnas de la matriz $\Phi$ y la matriz $\Psi$ como se muestra en la ecuación (4). En particular, se usa como una medida de calidad para la matriz $\Phi$, donde se busca que la coherencia sea muy pequeña lo que significa que su incoherencia será mayor y cumplirá esta propiedad:

$$
\mu(\Phi, \Psi)=\max \left|\Phi_{k} \Psi_{k}\right| .
$$

Las matrices más usadas y que cumplen estas propiedades son: las matrices gaussianas y binarias, aunque existen una gran cantidad de trabajos relacionados con matrices deterministas, no deterministas, adaptativas, no adaptativas, en las que se cumple con las propiedades citadas [4]. 
Por otra parte, es necesario desarrollar a la par un algoritmo de reconstrucción capaz de recuperar $x$ a partir de las mediciones $z$ dadas por la ecuación (2). Por lo tanto, un sistema de MC queda determinado por la toma de muestras $z$ de una señal $x$ a partir de una matriz $\Phi$ y una reconstrucción de $x$ a partir de las muestras $M$ tal que $M<N$.

En el caso especial de $M=N, x$ se recupera a partir de la inversa de $\Phi$, sin embargo, para el caso $M<N$, el sistema se vuelve indeterminado al existir un numero infinito de soluciones para $x$; sin embargo, si x cumple con las propiedades de dispersión e incoherencia la solución más dispersa es la solución correcta.

Una vez realizado el proceso de adquisición, se obtiene una estimación de la señal mediante un algoritmo de reconstrucción. Un enfoque práctico utilizado para determinar la solución dispersa es resolver este problema como un problema optimización convexa. Los trabajos originales versan sobre la minimización de la norma $l_{1}$ y la programación lineal, como se aprecia en la ecuación (5), la señal se reconstruye usando el problema de optimización [3,4]:

$$
\min \|\hat{x}\|_{1} \text { sujeto a } z=\Phi \Psi \alpha,
$$

donde: $\|\cdot\|_{1}$ denota la norma $l_{1}$ de un vector disperso, $\Psi$ es la matriz de dispersión, $\Phi$ es la matriz de muestreo y $\alpha$ son los coeficientes dispersos, de tal forma que al recuperar la señal se tiene $z=\Phi \widehat{x}$ donde $\widehat{x}$ es la solución óptima.

En el caso de los algoritmos de reconstrucción podemos mencionar una amplia gama de técnicas que incluyen: Búsqueda de Correspondencia Ortogonal (Orthogonal Matching Pursuit), Búsqueda de Base (Basis Pursuit) [4] entre otros, el método se selecciona dependiendo de la aplicación, el rendimiento, el tiempo y las necesidades de velocidad es importante mencionar que cada uno de estos métodos debe realizar un numero de iteraciones determinado para recuperar la señal de interés.

\section{Propiedades del muestreo compresivo aplicadas al electrocardiograma}

Esta sección comprende los conceptos que intervienen y están relacionados con las señales de ECG y el MC. Como se mencionó en las secciones anteriores el MC se fundamenta en dos propiedades: la dispersión, propiedad que tiene que ver directamente con la señal de interés y la incoherencia propiedad que hace referencia a la matriz de muestreo.

Dispersión de la señal de ECG. El grado de dispersión es proporcional al número de componentes cero presentes en la señal, de tal forma que a mayor número de componentes cero, mayor dispersión y viceversa, así, si observamos detenidamente una señal de ECG, Figura 1, podemos apreciar que, en el dominio del tiempo, esta presenta muy poca dispersión, en general, una señal de ECG por sí sola no es dispersa, y si mencionamos que el éxito del MC está directamente relacionado con la forma en la cual se representa nuestra señal, es de suma importancia representarla en un dominio distinto de manera que esta sea dispersa.

Debido a lo anterior, la mayoría de los trabajos sobre MC y ECG representan a la señal de ECG a partir de la Transformada Discreta de Fourier (FFT), la Transformada 


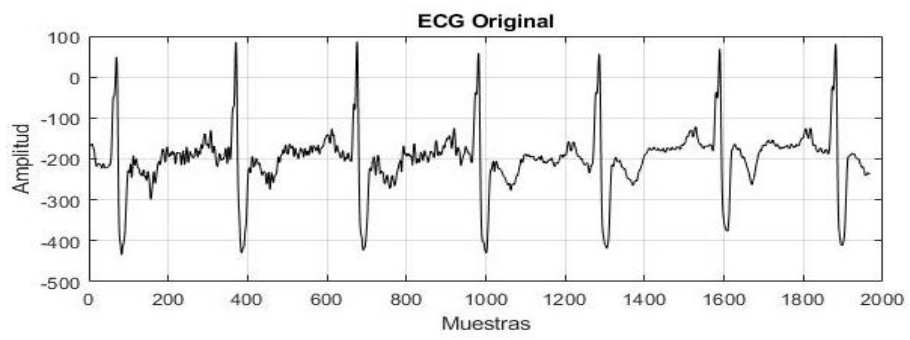

Fig. 1. Señal de Electrocardiograma.

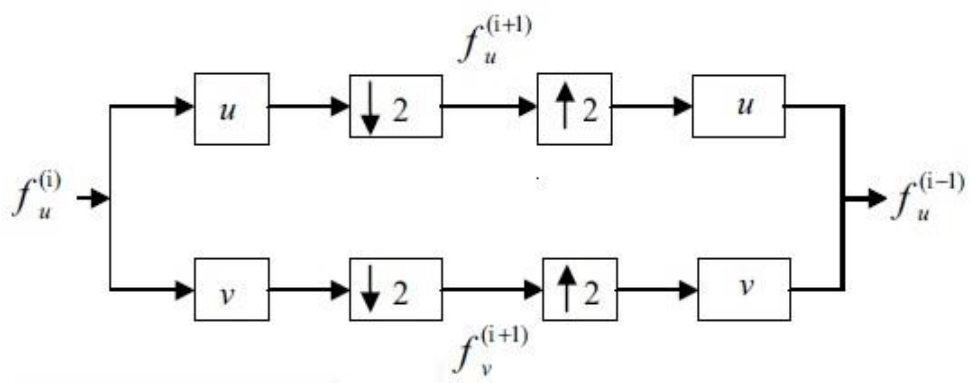

Fig. 2. Diagrama de Bloques de la DWT diádica.

Discreta de Coseno (DCT) o la Transformada Discreta de Wavelet (DWT) entre otros Finalmente esta última, la DWT es la que tiene el mayor auge dentro del MC debido a las propiedades intrínsecas de multi-resolución y compactación de energía [6]. Así, la DWT usada en nuestro método consiste en la descomposición de una señal con longitud $\mathrm{N}$ en $\log _{2} N$ niveles; llevada a través de un proceso de filtrado en serie y decimado para su compresión, por lo que para la reconstrucción de la señal original se interpola, se filtra en serie y se añaden sub-bandas, la Figura 2 muestra el diagrama de bloques para ambos procesos [10].

Cabe mencionar que la señal de entrada es filtrada usando un filtro paso-bajas $(u)$ para obtener los coeficientes de aproximación y un filtro paso-altas $(v)$ para los coeficientes de detalle, el proceso de filtrado se consigue por medio de la convolución entre los coeficientes del filtro y la señal de entrada, para conseguir una reconstrucción perfecta estos filtros deben de ser ortogonales [10]. Así los coeficientes de detalle nos dan información sobre la frecuencia baja y los de aproximación sobre la frecuencia alta, comúnmente para dispersión la frecuencia baja es de mayor importancia que la frecuencia alta por lo que los coeficientes de aproximación son siempre la entrada de los siguientes niveles de descomposición, lo que nos permite descartarlos.

De esta forma dada una señal $x$ de longitud $N$ y los filtros paso bajas (u) y paso altas $(v)$, los niveles de descomposición quedan representados por las ecuaciones (6) y (7), mientras que el proceso de reconstrucción se obtiene por medio de la ecuación (8). [10]: 


$$
\begin{aligned}
f_{u}^{(i)}(j) & =\sum_{k=1}^{2^{n-i+1}} u(k-2 j) f_{u}^{(i-1)}(k) \quad j=1,2, \ldots, 2^{n-i}, \\
f_{v}^{(i)}(j) & =\sum_{\substack{k=1 \\
2^{n-i+1}}} v(k-2 j) f_{v}^{(i-1)}(k) \quad j=1,2, \ldots, 2^{n-i}, \\
f_{u}^{(i-1)}(j) & =\sum_{k=1}^{2^{n-i+1}} u(j-2 k) f_{u}^{(i)}(k)+\sum_{k=1}^{2^{n-i+1}} v(j-2 k) f_{v}^{(i)}(k) .
\end{aligned}
$$

Matriz de muestreo. La matriz de muestreo tiene un papel importante, debido a que es la encargada de llevar a cabo las mediciones correctas para que sea posible la reconstrucción de la señal de ECG en el proceso siguiente, por lo que varios autores con el fin de cumplir con los criterios de incoherencia e isometría reservada han propuesto una serie de matrices para ECG como lo son las ampliamente utilizadas matrices aleatorias (Gaussianas o Binarias) [13], mientras que otros autores proponen matrices estructuradas como Toeplitz [2], circulares, o determinísticas [9].

Matriz transformada de Coseno. Esta matriz de $N x N$ es ampliamente utilizada en el procesamiento de imágenes en bloques pequeños [17], y puede expresarse de la siguiente forma:

$$
\Phi=\left\{\begin{array}{cll}
\frac{1}{\sqrt{M}} & p=0, & 0 \leq q \leq M-1, \\
\sqrt{\frac{2}{M} \cos \frac{\pi(2 q+1) p}{2 M}} & 1 \leq p \leq M-1, & 0 \leq q \leq M-1 .
\end{array}\right.
$$

De esta forma para una matriz $A$ de $N x N$ la operación $\Phi * A$ da como resultado una matriz de $N x N$ en la que cada columna contiene la DCT de una dimensión de las columnas de A. Cabe mencionar que al ser $\Phi$ ortogonal su inversa es la misma que su traspuesta.

Medidas utilizadas. Cualquier algoritmo de compresión debe tener la habilidad de preservar la calidad de la señal una vez que esta es descomprimida, aunado a esto, se debe contar con un buen radio de compresión, aunque lo único realmente importante es la calidad, pues es posible tener compresiones muy grandes con una pérdida de información proporcional. Así, en la compresión de ECG se debe considerar dos factores:

1. Eficiencia de compresión la cual puede ser medida con el radio de compresión (CR) por medio de la ecuación (10); donde $b_{o}$ hace referencia a los bits presentes en la señal de ECG original y $b_{c}$ a los bits de la señal comprimida, para nuestro caso se usa $b_{o}=2000$ y $b_{c}=312$, siendo $C R=6.4$.

$$
C R=\frac{b_{o}}{b_{c}}
$$

2. Calidad del esquema de compresión, que para esquemas con pérdida está determinada por la comparación entre los datos descomprimidos y los datos 


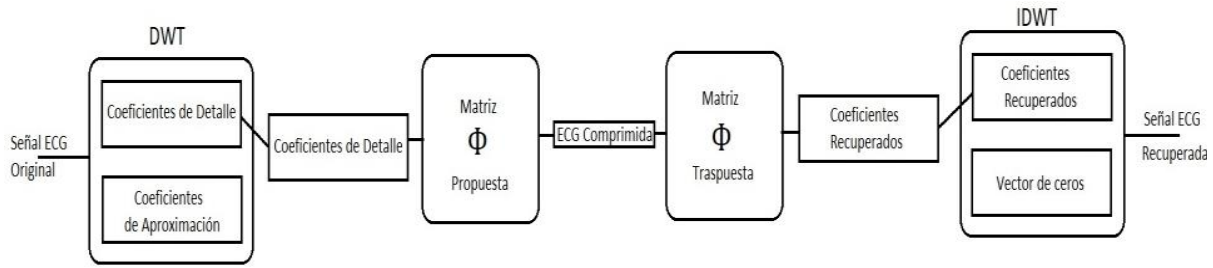

Fig. 3. Diagrama de bloques del algoritmo de compresión propuesto.

originales. Si no existen diferencias la compresión es sin pérdida, mientras que las medidas convencionales están basadas en distorsión, como lo son: el porcentaje de diferencia cuadrático medio (PRD), ecuación (11); y el porcentaje de diferencia cuadrático medio normalizado (PRDN) ecuación (12) [20]:

$$
\begin{gathered}
P R D=\frac{\|x-\hat{x}\|_{2}}{\|x\|_{2}}, \\
P R D N=\frac{\|x-\hat{x}\|_{2}}{\|x-\bar{x} e\|_{2}},
\end{gathered}
$$

donde para ambas ecuaciones $x$ y $\hat{x}$ representan a la señal original y a la señal recuperada respectivamente, en el caso de la ecuación (12) e representa un vector n-dimensional de unos y $\bar{x}$ representa el valor promedio de $x$.

\section{Algoritmo propuesto}

El diagrama de bloques del método de compresión propuesto es presentado en la Figura 3, El proceso inicia con una transformación lineal seguida del descarte de los coeficientes de aproximación y nuevamente una transformación lineal con la matriz de coseno para obtener los datos comprimidos del ECG. Los detalles de su implementación se detallan a continuación.

Dispersión propuesta de la señal de ECG. El proceso inicia con el procesado de la señal de ECG en tramas de $N$ muestras sin traslape, denotando $x$ como el vector de ECG $N$ dimensional que corresponde a la trama seleccionada y con $x \in R^{N}$, se dispersa la señal a través de una transformación lineal, en la cual se utiliza la DWT con una base ortonormal de Symlet, $\Psi=\left[\Psi_{1}, \Psi_{2} \cdots \Psi_{N}\right]$. Donde $\Psi$ es la representación matemática de la estructura de filtros mencionados en las ecuaciones (6) y (7) obteniéndose una representación conjunta de la señal en términos de los coeficientes de detalle $u$, y los coeficientes de aproximación $v$, así podemos denotar $\alpha=\Psi_{\mathrm{x}}$ donde $\alpha=\left[\begin{array}{l}u \\ v\end{array}\right]$ es la señal dispersa ECG con ambos coeficientes [11] 


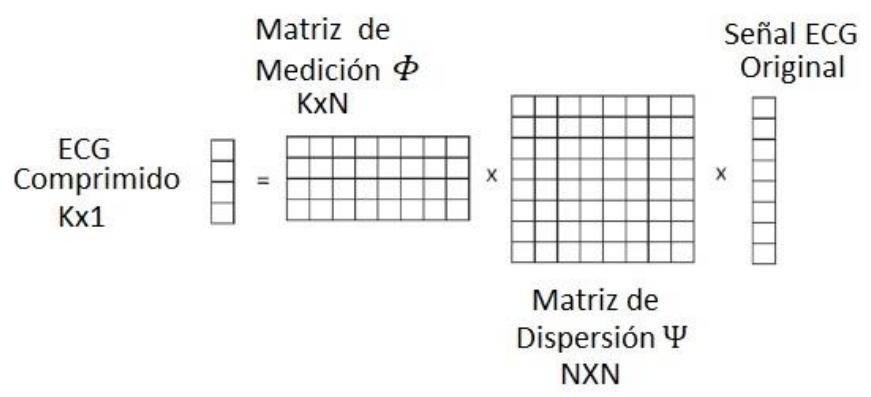

Fig. 4. Estructura matricial del esquema propuesto.

Tabla 1. Contraste de PRD Y PRDN.

\begin{tabular}{cccccccc}
\hline & \multicolumn{2}{c}{$\begin{array}{c}\text { Método } \\
\text { Propuesto }\end{array}$} & & \multicolumn{4}{c}{$\begin{array}{c}\text { Método de } \\
\text { Polania et al 2015[20] }\end{array}$} \\
\hline & \multicolumn{2}{c}{ MMO } & & \multicolumn{2}{c}{ MMB-IHT } & \multicolumn{2}{c}{ MMB-CoSaMP } \\
\hline Señal & CR & PRD & PRDN & PRD & PRDN & PRD & PRDN \\
\hline $\mathbf{1 1 5}$ & 6.4 & 0.6816 & 0.9876 & 2.74 & 6.62 & 2.57 & 6.19 \\
\hline $\mathbf{1 1 8}$ & 6.4 & 0.5879 & 1.0054 & 2.54 & 4.61 & 2.86 & 5.2 \\
\hline $\mathbf{1 1 9}$ & 6.4 & 0.5531 & 0.8109 & 2.29 & 4.5 & 2.61 & 5.12 \\
\hline
\end{tabular}

Tabla 2. Relación de Coherencia entre la señal y la matriz de medición.

\begin{tabular}{cc}
\hline Señal & Coherencia \\
\hline 115 & 0.0012 \\
\hline 118 & 0.0009 \\
\hline 119 & 0.0042
\end{tabular}

Matriz de muestreo. Una vez que se ha obtenido nuestra señal dispersa, $\boldsymbol{\alpha}=\boldsymbol{\Psi} \mathbf{x}$ se procede a solo tomar los coeficientes de detalle y descartar los coeficientes de aproximación, los cuales presentan una dimensión de $\mathbf{N} / \mathbf{4}$ siendo $\boldsymbol{N}$ la dimensión de nuestra señal original de ECG.

De notando a $\alpha_{d}$ como los coeficientes de detalle de nuestra señal de ECG se procede a construir la matriz de muestreo en base a la matriz de DCT de la ecuación (9).

$$
\Phi=\left\{\begin{array}{cll}
\frac{1}{\sqrt{L}} & S=0, & 0 \leq W \leq L-1 \\
\sqrt{\frac{2}{L} \cos \frac{\pi(2 W+1) S}{2 L}} & 1 \leq S \leq L-1, & 0 \leq W \leq L-1
\end{array},\right.
$$

donde $\mathrm{L}$ es la dimensión dada por los coeficientes de detalle $\alpha_{d}$, S y W son matrices compuestas de la siguiente forma:

$$
S=\left[\begin{array}{ccc}
0 & \cdots & 0 \\
\vdots & \ddots & \vdots \\
L & \cdots & L
\end{array}\right] ; W=\left[\begin{array}{ccc}
0 & \cdots & L \\
\vdots & \ddots & \vdots \\
0 & \cdots & L
\end{array}\right]
$$



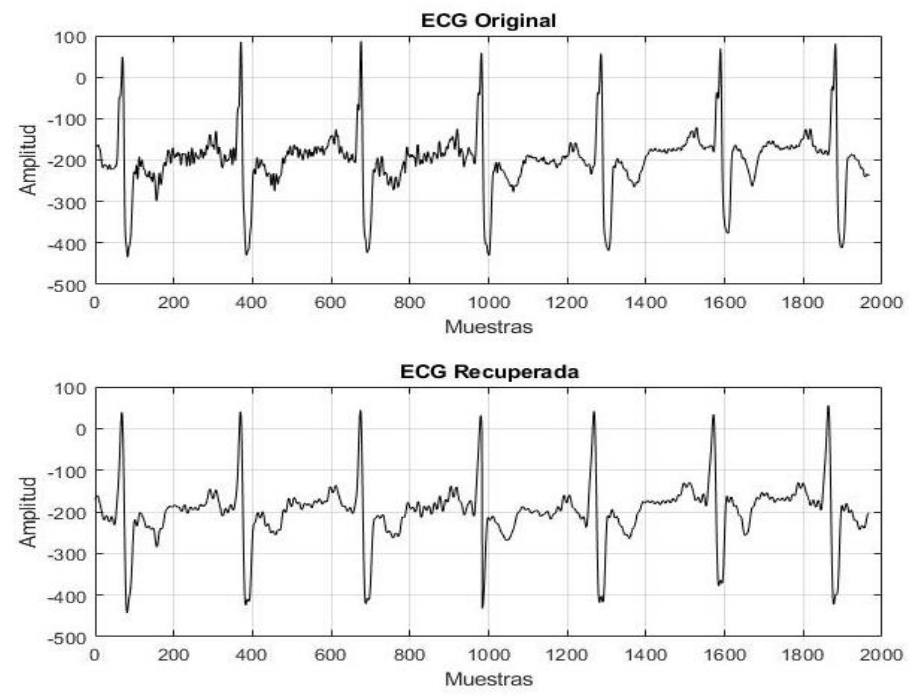

Fig. 5. Señal 118 de 2000 muestras original y recuperada.
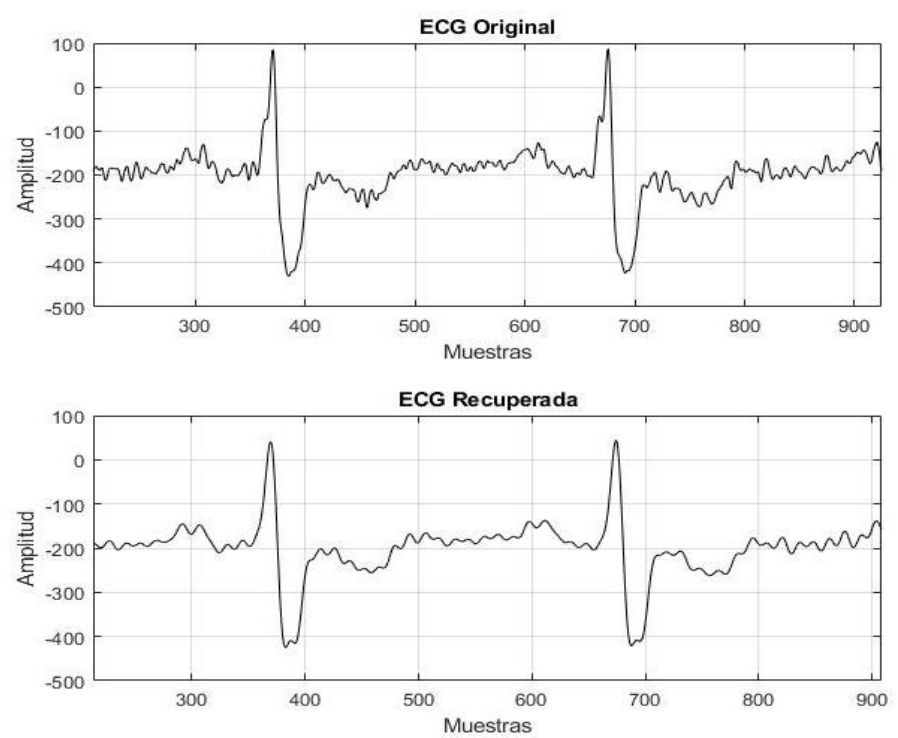

Fig. 6. Segmentos de la señal 118 de 2000 muestras original y recuperada.

Una vez construida la matriz mostrada en la ecuación (13), se procede nuevamente a realizar una transformación lineal de la forma $z=\Phi \alpha_{d}$ donde los renglones de la matriz $\Phi$ serán modificados de acuerdo con el nivel de compresión que se requiera. El proceso de transformación se muestra en la Figura 4. Debido a que una de las propiedades implícitas en la matriz DCT es la ortogonalidad, sabemos que la inversa de $\Phi$ es la traspuesta de $\Phi$.

Por lo que el proceso de reconstrucción se da a través de la aplicación de la matriz traspuesta $\Phi$ a las mediciones $y$,que contienen la señal de ECG comprimida, después 
se aplica la Transformada Inversa de Wavelet (IDWT) con el vector de los coeficientes de aproximación en cero y finalmente se recupera nuestra señal de ECG.

\section{Resultados y discusión}

Para evaluar el algoritmo propuesto se usa la base de datos de MIT-BIH-Arrhythmia [15] como nuestras señales de ECG, cada señal de ECG tiene 11-bits de resolución sobre $10 \mathrm{mV}$ y esta muestreada a una frecuencia de $360 \mathrm{~Hz}$, los experimentos son llevados a cabo sobre los registros extraídos con una longitud de $10 \mathrm{~min}$, tomando una longitud de 2000 muestras, para una tasa de compresión de 6.4 , los registros utilizados son $115,118,119$.

La forma de procesar cada ECG es por medio del algoritmo descrito en la sección previa, para la evaluación se utilizan las métricas descritas en la sección 3.3. cabe mencionar que estas mediciones no son acordes para los ECG ya que solo reflejan la distorsión presente en la señal desde un criterio estadístico.

En la Tabla 1 se muestra los valores del PRD y PRDN para las tres señales presentadas a una tasa de compresión de 6.4, las cuales son contrastadas contra los valores obtenidos por [15] cabe mencionar que, aunque se tiene un mejor desempeño esta no es una medida óptima para evaluar el ECG debido a que este es para uso médico por lo que es necesaria la evaluación de un profesional de la salud.

En la Tabla 2 se muestra la coherencia entre la señal original de ECG y la matriz de medición, como se vio en las secciones anteriores: a menor coherencia se llega a una mejor reconstrucción pues cumple con la propiedad de isometría reservada.

Las Figuras 5,6 y 7 nos muestran las señales originales, así como las recuperadas, específicamente en los todos los casos se encuentra una señal recuperada inteligible, donde el médico es capaz de realizar un diagnóstico.
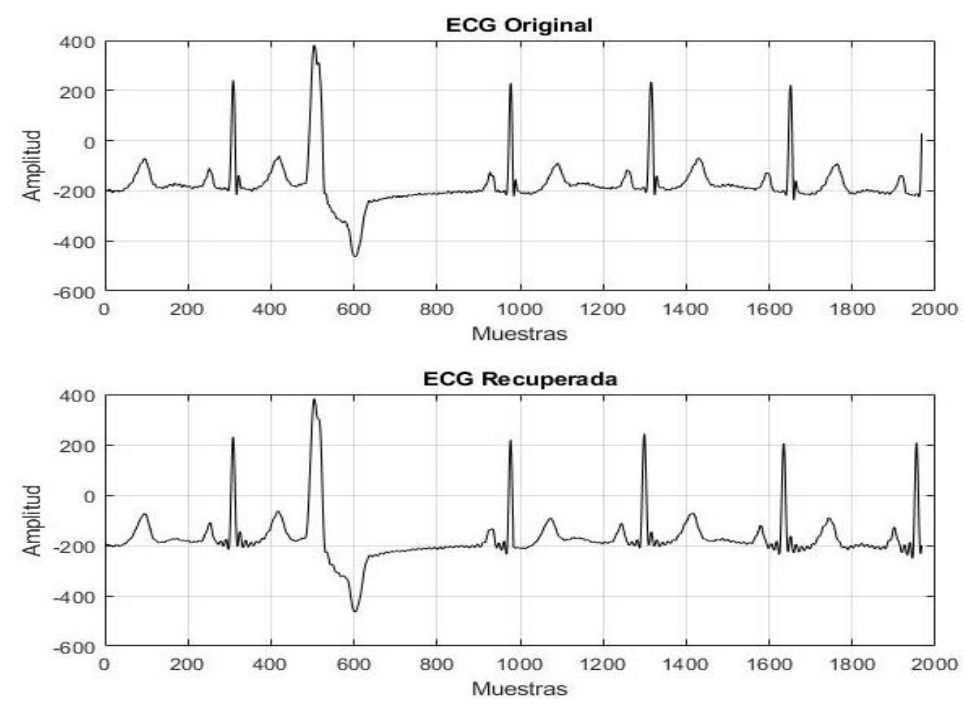

Fig. 7. Señal 119 de 2000 muestras original y recuperada. 
Con el fin de evaluar el desempeño del algoritmo se realiza un acercamiento para el caso de la señal 118 donde podemos ver que a pesar de no tener una etapa de reconstrucción con pocas muestras es posible recuperar inteligiblemente la señal de ECG.

Para señales más complejas el algoritmo muestra, de la misma forma que tiene un buen desempeño ya que es posible recuperarlas; en algunas partes la señal presenta una ligera distorsión, sin embargo, siguen siendo inteligibles para el profesional de la salud.

\section{Conclusiones}

El Algoritmo Propuesto cumple con las condiciones base de la teoría del muestreo compresivo, se efectúa la dispersión de la señal por medio de la función wavelet symlet, cumpliendo con la propiedad de dispersión, se cumplen con las propiedades de isometría reservada e incoherencia, al ser la coherencia de nuestra matriz cercana a cero como se aprecia en la Tabla 2. La matriz de medición es ortogonal lo que hace que el proceso de recuperación sea alcanzable con tan solo trasponer la matriz sin necesidad de utilizar algoritmos de reconstrucción los cuales tienen una mayor complejidad computacional y algunos de ellos son basados en iteraciones. El algoritmo propuesto es capaz de comprimir la señal hasta el punto de solo enviar pocos datos los cuales son codificados y recuperados por la misma matriz con una calidad inteligible para el profesional de la salud.

Agradecimientos. Este trabajo ha sido realizado con el apoyo del Consejo Nacional de Ciencia y Tecnología (CONACyT).

\section{Referencias}

1. Alwan, A.: Global Status Report on Noncommunicable Diseases 2010. World Health Organization, Geneva, Switzerland (2011)

2. Ahmed, N., Natarajan, T., Rao, K.R.: On image processing and a discrete cosine transform. IEEE Transactions on Computers, 23(1), pp. 90-93 (1974)

3. Candes, E., Romberg, J., Tao, T.: Robust Uncertainty Principles: Exact Signal Reconstruction from Highly Incomplete Frequency Information. IEEE Transactions on Information Theory, 52, pp. 489-509 (2006)

4. Candes, E.J., Wakin, M.B.: An Introduction to Compressive Sampling. IEEE Signal Processing Magazine, 25, pp. 21-30 (2008)

5. Chen, F., Chandrakasan, A.P., Stojanovic, V.M.: Design and Analysis of a HardwareEfficient Compressed Sensing Architecture for Data Compression in Wireless Sensors. IEEE Journal of Solid-State Circuits, 47, pp. 744-756 (2012)

6. Daubechies, I.: The wavelet transform, time-frequency localization and signal analysis. IEEE transactions on information theory, 36(5), pp. 961-1005 (1990)

7. Dixon, A.M.R., Allstot, E.G., Gangopadhyay, D., Allstot, D.J.: Compressed Sensing System Considerations for ECG and EMG Wireless Biosensors. IEEE Transactions on Biomedical Circuits and Systems, 6, pp. 156-166 (2012)

8. Donoho, D.L.: Compressed Sensing. IEEE Transactions on Information Theory, 52, pp. 1289-1306 (2006) 
9. Haupt, J., Bajwa, W.U., Raz, G., Nowak, R.: Toeplitz compressed sensing matrices with applications to sparse channel estimation. IEEE Transactions on Information Theory, 56, pp. 5862-5875 (2010)

10. Iwen, M. A.: Compressed sensing with sparse binary matrices: Instance optimal error guarantees in near optimal time. Journal of Complexity, 30(1), pp. 1-15 (2014)

11. Yan, J.: Wavelet Matrix. http://www.ece.uvic.ca/ jyan/ (2018)

12. Kumar, A., Kumar, G., Singh, K.: Electrocardiogram signal compression using singular coefficient truncation and wavelet coefficient coding. IET Science, Measurement \& Technology, 10(4), pp. 266-274 (2016)

13. Lorne, A., Stephen, D. H., Stephen, S., Calderbank, R.: Chirp sensing codes: Deterministic compressed sensing measurements for fast recovery. Applied and Computational Harmonic Analysis, 26, pp. 283-290 (2009)

14. Mamaghanian, H., Khaled, N., Atienza, D., Vandergheynst, P.: Compressed Sensing for Real-Time Energy- Efficient ECG Compression on Wireless Body Sensor Nodes. IEEE Transactions on Biomedical Engineering, 58, pp. 456-2466 (2011)

15. Moody, G.B., Mark, R.G.: The impact of the MIT-BIH Arrhythmia Database. IEEE Eng. Med. Biol. Mag. 20, pp. 45-50 (2001)

16. Polania, L.F., Carrillo, R.E., Blanco-Velasco, M., Barner, K.E.: Compressive Sensing Exploiting Wavelet Domain Dependencies for ECG Compression. In: Proceedings of the SPIE Defense, Security, and Sensing, Baltimore, pp. 23-27 (2012)

17. Polania, L.F., Carrillo, R.E., Blanco-Velasco, M., Barner, K.E.: On Exploiting Interbeat Correlation in Compressive Sensing-Based ECG Compression. In: Proceedings of the SPIE Defense, Security, and Sensing, Baltimore, pp. 23-27 (2012)

18. Polania, L.F., Carrillo, R.E., Blanco-Velasco, M., Barner, K.E.: Compressive Sensing for ECG Signals in the Presence of Electromyography Noise. In: Proceedings of the 38th Annual Northeast Bioengineering Conference (NEBEC), Philadelphia, pp. 16-18 (2012)

19. Polania, L.F., Carrillo, R.E., Blanco-Velasco, M., Barner, K. E.: Matrix completion-based ECG compression. In: Annual International Conference of the IEEE Engineering in Medicine and Biology Society, Boston, MA, pp. 1757-1760 (2011)

20. Polanía, L, Carrillo, R.E., Blanco-Velasco, M., Barner, K. E.: Exploiting prior knowledge in compressed sensing wireless ECG systems. IEEE Journal of Biomedical Health Informatics, 19(2), pp. 508-519 (2015)

21. Zhang, Z., Jung, T., Makeig, S., Rao, B.D.: Compressed Sensing for Energy-Efficient Wireless Telemonitoring of Non-Invasive Fetal ECG via Block Sparse Bayesian Learning. IEEE Transactions on Biomedical Engineering, 60(2), pp. 300-309 (2013) 